Document downloaded from:

http://hdl.handle.net/10251/64592

This paper must be cited as:

Balador, A.; Tavares De Araujo Cesariny Calafate, CM.; Cano Escribá, JC.; Manzoni, P. (2015). DTB-MAC: Dynamic Token-Based MAC Protocol for reliable and efficient beacon broadcasting in VANETs. 12th IEEE Consumer Communications and Networking Conference (CCNC 2015). IEEE. doi:10.1109/CCNC.2015.7157955.

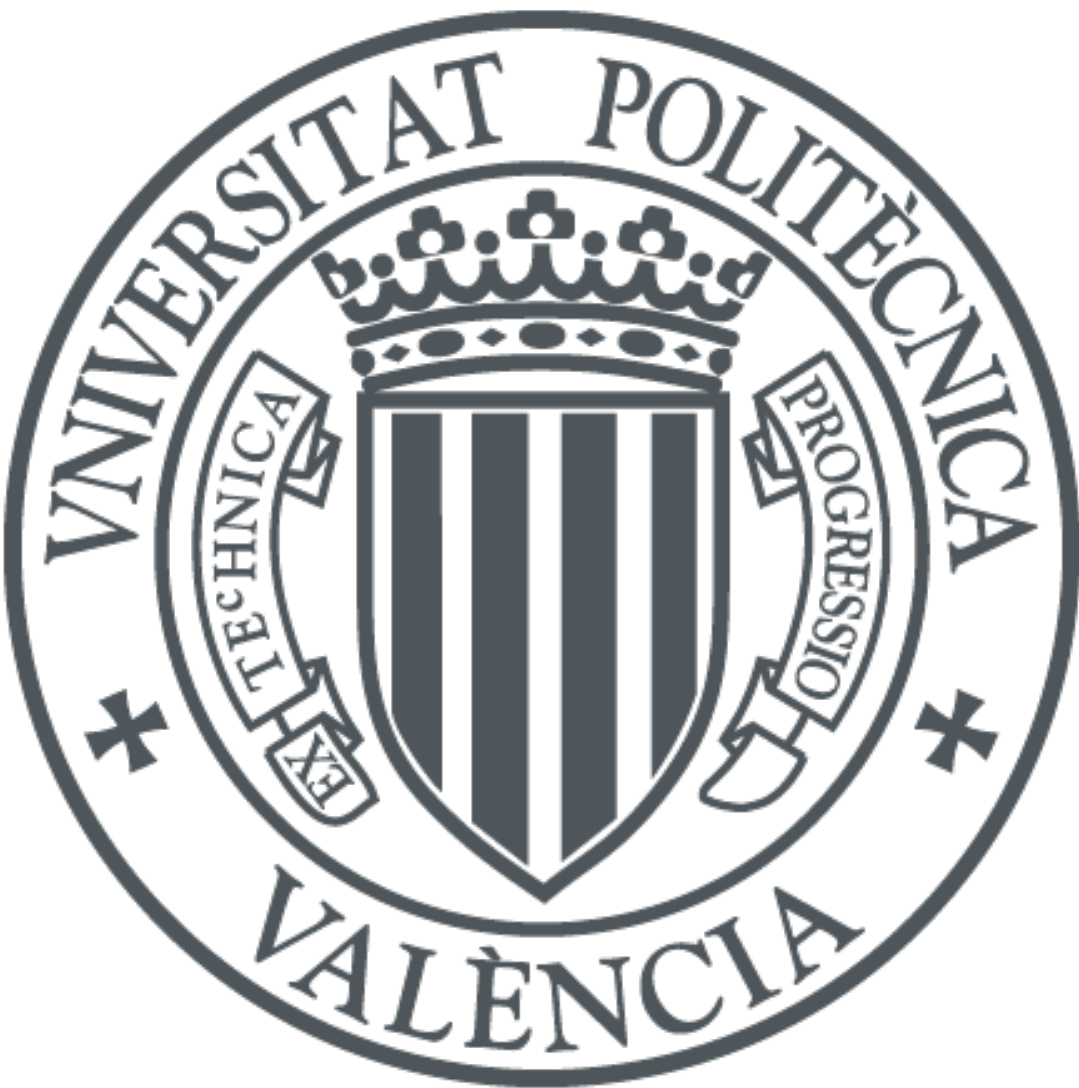

The final publication is available at

http://dx.doi.org/10.1109/CCNC.2015.7157955

Copyright IEEE

Additional Information

(C) 2015 IEEE. Personal use of this material is permitted. Permission from IEEE must be obtained for all other uses, in any current or future media, including reprinting/republishing this material for advertising or promotional purposes, creating new collective works, for resale or redistribution to servers or lists, or reuse of any copyrighted component of this work in other works. 


\title{
DTB-MAC: Dynamic Token-Based MAC Protocol for Reliable and Efficient Beacon Broadcasting in VANETs
}

\author{
Ali Balador, Carlos T. Calafate, Juan-Carlos Cano and Pietro Manzoni \\ Universitat Politecnica de Valencia \\ Camino de Vera, s/n, 46022 Valencia, Spain \\ Email: alba6@upv.es, calafate, jucano,pmanzoni@disca.upv.es
}

\begin{abstract}
Most applications developed for vehicular environments rely on broadcasting as the main mechanism to disseminate their messages. However, in IEEE 802.11p, which is the most widely accepted MAC protocol for vehicular communications, all transmissions remain unacknowledged if broadcasting is used. Furthermore, safety message transmission requires a strict delay limit and a high reliability, which is an issue for random access MAC protocols like IEEE 802.11p. Therefore, transmission reliability becomes the most important issue for broadcast-based services in vehicular environments. In this paper, we propose a hybrid MAC protocol, referred as Dynamic Token-Based MAC Protocol (DTB-MAC). DTB-MAC uses both a token passing mechanism and a random access MAC protocol to prevent channel contention as much as possible, and to improve the reliability of safety message transmissions. Our proposed protocol tries to select the best neighbouring node as the next transmitter, and when it is not possible, or when it causes a high overhead, the random access MAC protocol is used instead. Based on simulation experiments, we show that the DTB-MAC protocol can achieve better performance compared with IEEE802.11p in terms of channel utilization and beacon delivery ratio.
\end{abstract}

Keywords-vehicular ad hoc network (VANET), IEEE802.11p, beacon broadcasting, token Passing, clustering, reliability.

\section{INTRODUCTION}

The high number of people who die in car crashes, along with the increasing time spent in vehicle traffic congestions, has led to a gradual transition towards Intelligent Transportation Systems (ITS) and related applications, which aim at improving road safety or provide smart navigation or ecofriendly applications. Vehicular ad hoc networks (VANETs) provide a communication structure for ITS by equipping cars with advanced sensors and communication devices that enable a direct exchange of information between vehicles.

Safety applications represent the main target of VANET communications. Their goal is to increase each vehicle's awareness about its neighbourhood through message broadcasting in order to reduce the number of car accidents. For this purpose, they use two types of messages which are called: Cooperative Awareness Messages (CAM) or beacon, and Decentralised Environment Notification (DEN) or emergency messages [1]. Beacons are sent periodically and include information such as geographical location, speed, and acceleration. Beacons are very small, only sent to a close neighbourhood, and the validity of the information they contain is very limited in time. Emergency messages are only generated in emergency situations such as sudden lane-changes or precrash situations. In this paper, we focus on single-hop periodic safety message broadcasting.

The reliability of safety message transmissions is the main issue for vehicular communications because the safety of people on the road is directly related to the effectiveness of these transmissions. A VANET MAC protocol must guarantee reliable beacon broadcasting within deadline bounds to all vehicles in the neighbourhood, in order to notify them about unsafe driving conditions. However, high node mobility, highly dynamic topology, and lack of a central control unit, make the design of a reliable MAC protocol for vehicular environments a very difficult task, especially when focusing on message broadcasting.

IEEE802.11p [2] proposes an amendment to the existing IEEE 802.11 standard for operation in the dedicated shortrange communications (DSRC) [3] band. It has been widely reported in literature [4], [5], [6] that IEEE802.11p cannot prevent neighbouring vehicles from simultaneously transmitting at high densities. Therefore, it is prone to cause collisions and long delays between successful packet receptions. In American and European standards, safety messages are generated every $0.1 \mathrm{~s}$ or at even higher rates for applications that require a high level of safety, which makes the channel very busy. Moreover, there are no acknowledgements or RTS/CTS (Readyto-Send/Clear-to-Send) frames, which causes IEEE802.11p to behave similarly to an ALOHA MAC protocol in broadcast mode. Therefore, it becomes more difficult to achieve a high broadcast reliability due to the channel contention and interferences.

Although many new broadcasting schemes have been proposed for VANETs, they are not compatible with different vehicular environments, usually failing to encompass both small and high vehicle densities. For example, time division multiple access (TDMA) based techniques have been recently proposed to improve broadcasting reliability in vehicular networks. However, the wasted time associated to the usage of fixed length frames prevents such methods from becoming an efficient choice for low density scenarios. Moreover, the probability that different vehicles choose a same slot can be mentioned as another obstacle for the adoption of these methods under heterogeneous VANET scenarios.

In this paper, we propose a method that combines both random access with token passing techniques to propose a 
new low-overhead token-based MAC protocol for VANETs. In particular, our token passing mechanism was implemented on top of a random access MAC protocol, being especially useful under high densities, a situation where the $802.11 \mathrm{p}$ protocol usually fails to achieve a good performance. In order to provide better reliability than IEEE 802.11 p, each node gives the privilege to transmit to another node in its neighbourhood whenever possible, and therefore it can improve throughput of VANETs.

The rest of the paper is organized as follows. Section II outlines the related work on beacon broadcasting. In section III, we describe the new proposed token-based scheme in detail. Section IV describes the simulation scenarios, the measurements, and the selected metrics for performance evaluation. Performance evaluation results are presented in section V, including a comparison of our protocol against IEEE 802.11p in a highway scenario. Finally, section VI concludes this paper.

\section{RELATED WORKS}

Due to the inherent drawbacks of CSMA-based MAC protocols, they cannot be a good choice for low-latency safety applications in VANETs. To address this problem, time division multiple access (TDMA) and space division multiple access (SDMA) based techniques [7], [8], [9], [10], [6] have recently received much attention in the VANET literature because they are able to provide guaranteed delay. TDMAbased schemes rely on assigning different time slots to the vehicles that are closer to each other in order to minimize the contention chances among vehicles, reusing the same slot times for farthest vehicles. A common problem of TDMAbased mechanisms is that they use fixed length frames, and so two or more vehicles can reserve the same slot. Although some new modifications have been proposed to solve these problems and make such approaches more compatible with vehicular environments, they still require additional efforts.

In SDMA-based techniques [9], [10], the road is divided into subsets which are called cells. A particular set of time slots are assigned to each cell, so that each vehicle chooses its time slot based on its location on the road. The main problem of SDMA-based schemes is time scheduling complexity; in addition, their resource utilization efficiency decreases in VANETs due to unbalanced traffic density and dynamic topology changes.

The effectiveness of these two types of MAC protocols have been already compared by several authors [6], [11], [12], [13], [14]. The overall results show that, while TDMA-based solutions (particularly STDMA) obtain better results in terms of fairness and delay, they do not show a good packet delivery probability under high vehicle densities. Also, the implementation of TDMA-based approaches is quite complicated.

The token ring method can be implemented on top of IEEE 802.11 to offer QoS provisioning in terms of reserved bandwidth and bounded delay when operating under high densities. The wireless token ring protocol (WTRP) [15] was the first scheme using this idea in vehicular environments. However, it is still incapable of adapting to the fast topology changes typical of these environments. Some modifications were proposed in order to solve this issue: in [16], a wireless dynamic token protocol (WDTP) is presented which defines different subsets of vehicles and, in each subset, there is a master node responsible for token management. In [17], a token-based scheduling scheme is presented where vehicles don't have to maintain an ordered list of their neighbour nodes, and where each vehicle stochastically passes the token to others. Nevertheless, the authors assumed that the network must be fully-connected, which is not the case in VANETs.

In [18], an overlay token ring protocol (OTRP) is presented for vehicular environments. It operates in two modes: in the ordinary mode, beacons circulate among the neighbouring nodes; the other mode is used in case of an accident. By adapting the token passing solution and using different modes, OTRP achieves better performance and rapid emergency message delivery in VANET environments. However, the authors did not consider some issues which are very challenging in VANETs, such as interferences among different rings. Also, the simulation environment was restricted to only a few nodes, and they used a proprietary $\mathrm{C}++$ based simulator that prevents the research community from doing a thorough validation. In [19], a multi-channel token ring MAC protocol (MCTRP) is presented for inter-vehicle communications. In MCTRP, vehicles are grouped into rings based on their speed. Since this protocol has to make groups and central vehicle election very frequently, it introduces a high overhead, which makes it unsuitable for high-speed networks.

From a global perspective, we find that the main challenge in designing a dynamic token-based MAC protocol for vehicular environments is reducing of the ring control overhead. As we have mentioned before, token based methods are able to support QoS provisioning, but the high number of nodes and the rapid topology changes typical of vehicular environments are prone to introduce a high overhead. In order to reduce token-based overhead, and make our solution adaptable to different network densities, we propose combining a random access MAC protocol and a token passing solution. Our proposed protocol (DTB-MAC) chooses one node from the neighbourhood and gives it the privilege to broadcast either CAM or DEN messages, thereby reducing the contention overhead and improving reliability as much as possible. Whenever the DTB-MAC protocol finds a problem to circulate the token, it uses a random access MAC protocol to provide a suitable situation to switch back again to the token passing routine.

\section{DYNAMIC TOKEN-BASED MAC PROTOCOL} (DTB-MAC)

In this section, we present the DTB-MAC protocol, a novel solution targeting vehicular environments that combines the token passing concept with a random access MAC protocol in order to improve the reception ratio of broadcasted beacon messages.

We consider a vehicular environment with vehicles (the term "vehicle" and "node" are used interchangeably throughout this paper; the meanings of the most important symbols are summarized in Table I) which pertain to one or more virtual rings, as shown in Figure 1. We introduce the concept of "virtual rings", which are rings created between vehicles in the same neighbourhood that are dynamically defined based on the vehicle mobility. Each node starts as an individual entity, and transmits its beacon without cooperating with its surrounding 
environment. With the first beacon reception, a node is notified about the existence of other nearby nodes and tries to join available rings in its neighbourhood. All the nodes interested in joining a ring compete with other nodes through a random access MAC protocol. The main benefit of this strategy is that, after joining a ring, nodes no longer need to compete with each other to gain channel access, except in those cases where the token is lost.

Within the ring, only the node holding the token can transmit a frame (in our case a beacon) on the channel. The term "token" refers to a privilege which is given to a ring member when it is chosen by another ring member to be the next beacon transmitter. Notice that DTB-MAC does not require any extra packet transmission for token passing, being that nodes are notified about the next transmitter only by listening to the beacon transmission since a piggybacking approach is adopted. While the token is circulating in a ring, nodes can find their turns to access to the channel based on beacon reception. In particular, the token holder selects another ring member as the next token holder, and includes that information in the beacon being transmitted. If the token is lost for any reason, our solution relies on a random access MAC method in order to find a new transmitter and inject a new token into the ring, thereby keeping it alive. It is important to highlight that, by keeping the token circulating in the ring as much as possible, DTB-MAC is able to provide improved performance.

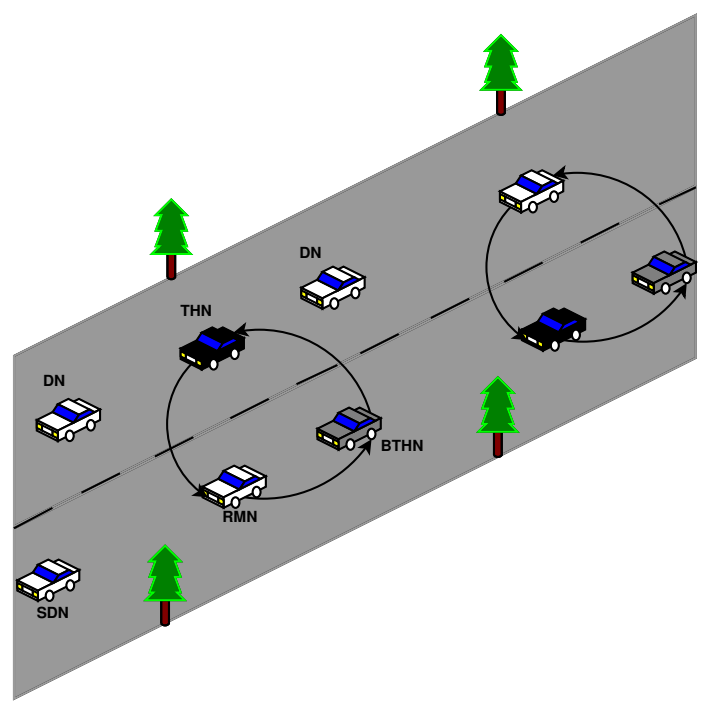

Fig. 1. Highway scenario.

As shown in Figure 1, vehicles in the system can be in the following states:

- Token Holder Node (THN): a node which is allowed to transmit.

- $\quad$ Backup Token Holder Node (BTHN): a node which is allowed to transmit if the THN node fails to transmit.

- $\quad$ Ring Member Node (RMN): a node which is in a ring but cannot transmit since it does not hold the token.

- Dissociative Node (DN): a node which does not belong to any ring and is not part of a ring joining procedure either.

- Semi-Dissociative Node (SDN): a node which does not belong to any ring, but it is attempting to join a ring following a beacon reception.

Figure 2 illustrates the transitions between these different states in the scope of our proposal. The key processes involved in the state transitions are described in more detail below.

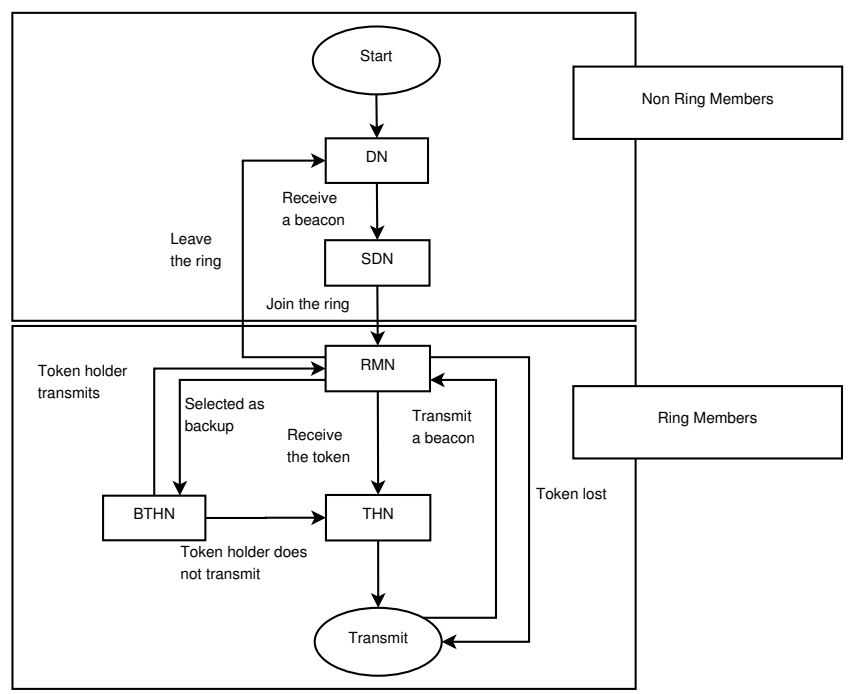

Fig. 2. State transition diagram of DTB-MAC.

\section{A. Joining Process}

Joining a ring is always an issue for token-based MAC protocols which are designed for dynamic wireless networks, especially in VANETs with very high dynamics. The complexity stems from the collision probability between the ring members and new nodes attempting to join the ring. The top part of Figure 2 shows the joining process in DTB-MAC; notice that each vehicle starts from the DN state. If no beacons are received, it remains in that state, meaning that it will belong to a single-node ring and generate its own beacons. Upon a beacon reception from neighbouring nodes, the node switches to the SDN state.

When we have more than one SDN node in one neighbourhood, these nodes will compete with each other in order to join the ring by sending their own beacon, meaning that there is a potential for collision occurrence. To reduce this problem, the nodes in the SDN state must wait for a predefined time period $\left(t_{T H N}\right)$, plus a dynamic time period $\left(t_{D I F F} \geq 0\right)$. A token holder chooses to transmit or to allow an SDN node to join a ring after time period $t_{T H N}$. Therefore, SDN nodes must wait at least $t_{T H N}$ after a beacon reception in order to avoid collision with the scheduled ring transmission. Also, by waiting for a $t_{D I F F}$ period, DTB-MAC, decreases the probability of collision occurrence among SDN nodes. The value of $t_{D I F F}$ 
TABLE I. SUMMARY OF IMPORTANT SYMBOLS

\begin{tabular}{ll}
\hline Symbol & Definition \\
\hline \hline THN & token holder node \\
BTHN & backup token holder node \\
RMN & ring member node \\
DN & dissociative node \\
SDN & semi-dissociative node \\
$t_{D N}$ & the waiting time for transmitting beacon by DN \\
$t_{T H N}$ & the waiting time for transmitting beacon by THN \\
$t_{B T H N}$ & the waiting time for transmitting beacon by BTHN \\
$t_{\text {old }}$ & an old neighbouring list entry will be deleted after this period \\
$p_{R M N}$ & the probability that one RMN transmit \\
$t_{j o i n}$ & the time period after which a THN sends if SDNs do not send \\
$t_{r e m}$ & remaining time until next beacon generation \\
$\mathrm{C}$ & a random number between 0 and $t_{\text {rem }}$ \\
$t_{D I F F}$ & a dynamic time period which is calculated based on C and Q \\
\hline \hline
\end{tabular}

is dynamically calculated based on the remaining time until the next beacon generation $\left(t_{r e m}\right)$ according to the following expression:

$$
t_{D I F F}=\alpha \times C
$$

where $\mathrm{C}$ is a random number of time slots between 0 and $t_{r e m}$, and $\alpha$ is a value between 0 and 1 that allows fine tuning the protocol behaviour in order to keep the DTB-MAC delay low. As we increase the value of $\alpha$, it decreases the number of collisions between SDN nodes. However, bigger $\alpha$ values decrease the probability of joining a ring. Therefore, $\alpha$ must make a balance between the probability of joining to the ring and the number of collisions between SDN nodes. $t_{D I F F}$ decreases with decreasing $t_{r e m}$ values. Whenever an SDN node finds the channel idle and transmits a beacon, it switches to the RMN state. As a result, the probability of collision occurrence decreases, although it cannot be reduced to zero.

\section{B. Ring Management}

As we mentioned before, the highly dynamic topology typical of vehicular environments difficults the creation of fixed clusters and selecting one of the ring members as a coordinator, being such options undesirable in general. Therefore, the VANET research community looks for decentralized methods in order to decrease the overhead associated to central element selection processes.

DTB-MAC does not need any coordinator to manage internal ring competitions for accessing the channel. Each node is responsible of keeping the token circulating inside the ring. Before explaining how DTB-MAC manages the token passing, we define the frame header format in both IEEE 802.11p and DTB-MAC protocols.

Figure 3 introduces the header format of the IEEE $802.11 \mathrm{p}$ and DTB-MAC protocols. The header used by DTB-MAC is an extension of IEEE $802.11 \mathrm{p}$ with some extra information. As can be seen in the figure, we only add one new field, $t_{r e m}$, which includes the remaining time before the next beacon generation. Address 3 and Address 4 fields are used in order to identify the token holder and the backup token holder nodes, respectively. The remaining fields have the same usage as in the IEEE $802.11 \mathrm{p}$ standard.

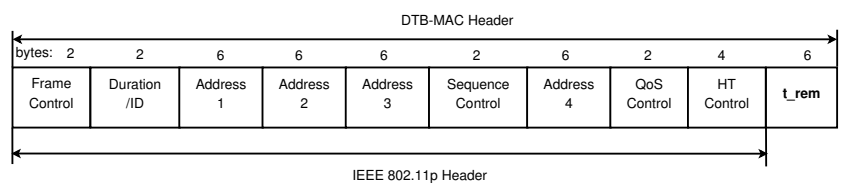

Fig. 3. IEEE $802.11 \mathrm{p}$ and DTB-MAC header format.

In order to maintain the token, ring members have three responsibilities:

1) Making a list of ring members: In order to grant another node in the ring the permission to transmit, each node must make a list of its neighbours based on data retrieved from its beacons. Whenever a node receives a beacon, it saves the source address and the $t_{r e m}$ of the beacon source in the neighbouring list. The neighbouring list is updated following every beacon reception. If a record related to the source node already exists in the list, only the $t_{r e m}$ value is updated. Otherwise, a new record is created for the source node.

2) Selecting next token holders: Before each beacon transmission, a node must select two nodes from its neighbouring list as the next token and the backup token holder, and also measure its own $t_{\text {rem }}$. This information is included in the beacon and broadcasted. Therefore, the neighbouring nodes can decide whether to send (THN) or wait (RMN and BTHN) based on the information received. THN and BTHN are selected based on $t_{r e m}$, meaning that each node selects two nodes from its neighbouring list with the lowest $t_{r e m}$. This mechanism assigns a higher priority to those nodes with lower $t_{\text {rem }}$ in order to allow them sending their beacons before reaching the deadline. We need to mention that expired beacons are dropped. Therefore, DTB-MAC will always have, at most, one safety message to transmit.

3) Recovering from a lost token: Ring members which are not selected as token holder or backup token holder check the channel during a predefined time period $t_{B T H N}$ (waiting for selected token and backup token holder transmissions). If they cannot detect any activity in the channel during this period, it means that a token loss has occurred. This situation can be due to a problem in the previous beacon reception, causing the selected nodes to miss their THN/BTHN status notification. Also, it can occur that they do not have any beacon in their queues to transmit at the time they receive the beacon. To solve 
this problem, and to regenerate the token, ring members wait for a time period equal to $t_{D I F F}$, as calculated in Equation 1. Then, one of the ring members (the one choosing the smallest $t_{D I F F}$ value) transmits and selects the next token holder.

\section{Ring Operation}

There are three possibilities for a ring member to transmit a beacon as shown in the bottom part of Figure 2 .

First, if a node is selected as the token holder, it switches to the THN state, and, following the beacon reception, the node chooses to transmit its own beacon with a probability $p_{R M N}$, after a time period $t_{T H N}$. If it chooses not to send after $t_{T H N}$, it delays the transmission for a predefined time period $\left(t_{\text {join }}\right)$ and then transmits only if it finds the channel idle (a new node does not join the ring). Second, if it is selected as a backup token holder, it changes its state to BTHN, and it transmits if it finds the channel idle for one slot time after time period $t_{B T H N}$. BTHN is calculated according to the following expression:

$$
t_{B T H N}=t_{T H N}+t_{j o i n}
$$

The third case happens after a token loss, so that the selected nodes (THN or BTHN) do not transmit. In that case, other ring members have the opportunity to transmit instead. If a node is not selected, it checks the channel during a $t_{B T H N}$ time period after the beacon reception (waiting for the selected token or backup token holder transmission). If it cannot detect any activity in the channel during this period, it waits for a time period equal to $t_{D I F F}$, as calculated in Equation 1 . Then, it transmits and regenerates the token, if it can find the channel free. Waiting for a dynamic time period, $t_{D I F F}$, causes a reduction on the number of collisions between RMN nodes. After transmission, each node goes back to the RMN state and waits until it again obtains the permission to transmit. Figure 4 determines the transmission order of the different nodes.

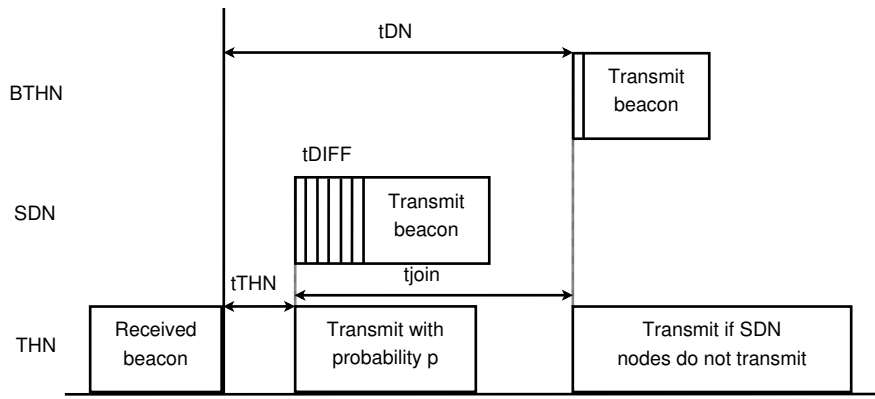

Fig. 4. Transmission scheduling in DTB-MAC.

\section{Leaving Process}

If ring members do not remove old entries, they could select nodes which are no longer in their neighbourhood. This situation would increase the delay and degrade the efficiency of DTB-MAC, thus being clearly undesirable. To avoid this, ring members remove old entries, meaning that each node removes the nodes in its neighbouring list if it fails to receive any beacons from them during a predefined time period $\left(t_{\text {old }}\right)$. This value was chosen based on topology change speed. If the topology changes very fast, the chosen value should be smaller in order to detect nodes leaving a ring as soon as possible. In this paper, we chose an optimised static parameter for the selected simulation scenario environment. However, for the future works, it should be dynamically chosen based on the nodes mobility to obtain the best performance in different network scenario environments.

\section{Simulation}

To assess the effectiveness of the proposed DTB-MAC protocol we rely on simulation. In this section, we describe the simulation details, including simulation scenario parameters and the protocol configurations.

We consider a highway scenario with $2.2 \mathrm{~km}$ length and 2 lanes, where the width of each lane is $3 \mathrm{~m}$. We used SUMO [20] in order to generate realistic vehicular mobility. Also, we implemented our proposed MAC protocol in OMNeT++ (version 4.4.1) [21] and used the IEEE802.11p implementation made available by the Veins framework (version 2.1) [22] for $\mathrm{OMNeT}++$. Table II summarizes the simulation parameters.

TABLE II. THE SIMULATION PARAMETERS

\begin{tabular}{ll}
\hline Simulation Parameter & Value \\
\hline \hline Simulation time & $280 \mathrm{~s}$ \\
Warm-up time & $250 \mathrm{~s}$ \\
Density & $16-43$ vehicles/lane/km \\
Highway Length & $2200 \mathrm{~m}$ \\
Number of lanes & 2 \\
Transmission Range & $500 \mathrm{~m}$ \\
Propagation model & Sommer et. al obstacle based model \\
& with Shadowing + Nakagami small \\
& scale fading \\
Packet arrival rate & 10 pkts/s \\
Packet length & $500 \mathrm{bytes}$ \\
Frequency & $5.9 \mathrm{GHZ}$ \\
Data Rate & $6 \mathrm{Mbps}$ \\
Beacon Priority & $\mathrm{AC}[0]$ \\
Broadcasting CW & 15 \\
\hline \hline
\end{tabular}

Each beacon includes 500 bytes payload and it is generated every $0.1 \mathrm{~s}$. Also, it is transmitted with a data rate of $6 \mathrm{Mbps}$ within a transmission range of $500 \mathrm{~m}$. Traffic density varies from 16 to 43 cars $/$ lane $/ \mathrm{km}$. We assumed that for each vehicle, acceleration $\left(\mathrm{m} / \mathrm{s}^{2}\right)$, declaration $\left(\mathrm{m} / \mathrm{s}^{2}\right)$, and the maximum speed $(\mathrm{m} / \mathrm{s})$ are set to 2,4 , and 50 , respectively. In order to present a more realistic vehicular environment, we used the radio propagation model made available by Veins for VANET communications [23]. We run the simulation for 280 seconds, although results are only captured after the 250 seconds to allow the system to reach a steady state. The beacon priority and the broadcasting $\mathrm{CW}$ are $\mathrm{AC}[0]$, and 15, respectively.

Table III shows the parameters which are used by the DTB-MAC protocol. These parameter values were achieved based on extensive simulations in which different combinations of values were evaluated to obtain the best performance in the selected simulation scenario. $t_{T H N}$ was obtained so that each beacon is received by all neighbouring nodes before a new beacon transmission. Also, $t_{\text {join }}$ is defined based on the average time period in which nodes can join a new ring.

According to [24], we chose widely used performance metrics to evaluate the performance of the proposed scheme: 
TABLE III. DTB-MAC PROTOCOL PARAMETERS

\begin{tabular}{ll}
\hline Parameter & Value \\
\hline \hline$t_{D N}$ & $0.9 \mathrm{~s}$ \\
$t_{T H N}$ & $0.25 \mathrm{~ms}$ \\
$t_{\text {old }}$ & $0.1 \mathrm{~s}$ \\
$t_{\text {join }}$ & $3 \mathrm{~ms}$ \\
$\alpha$ & 0.1 \\
\hline \hline
\end{tabular}

(a) Beacon Delivery Ratio (BDR), which is defined as the ratio between the number of beacons successfully received by nodes in the transmission range and the number of beacons transmitted; (b) average number of MAC collisions per second, which shows the average number of collisions experienced per second; (c) dropped beacon ratio, which is calculated as the number of beacons that are dropped because the beacon transmission deadline expires to the total number of beacons; and (d) channel utilization, which shows the amount of time that the channel is used for successful and failed transmissions, along with the, idle time.

\section{Result And AnAlysis}

To show that our proposed protocol (DTB-MAC) is able to boost performance, we first compare the beacon delivery ratio of DTB-MAC and IEEE 802.11p protocols under different network densities.

Figure 5 shows the results for different network densities. For the beacon delivery ratio calculation, we assumed zero delivery ratio for dropped packets. We noticed that, as expected, DTB-MAC increases the beacon delivery ratio for different network densities by decreasing the number of channel contentions. Since DTB-MAC can perfectly circulate the token between ring members, it can prevent collision occurrence. As can be seen, DTB-MAC achieves more improvements in higher than lower network densities. The reason is that, or lower densities, the number of nodes in each neighbourhood is low, which increases the probability of choosing a neighbour node without a beacon ready for transmission. This issue causes a lot of failures in the token passing procedure, causing DTB-MAC to switch to the random access MAC protocol. Therefore, the amount of improvement that DTB-MAC achieves at low densities differs from the high density case. In this way, DTB-MAC can solve the problem of TDMA-based techniques by providing a good beacon delivery ratio in high density networks, while it can also show improvement compared to IEEE $802.11 \mathrm{p}$ in low density networks.

To better understand how DTB-MAC is able to achieve higher beacon delivery ratios, in Figure 6 we present the average number of collisions per second for both DTB-MAC and IEEE 802.11p. This figure shows that, in low densities, DTB-MAC is not so effective at preventing collisions as at high densities. These results stem from the fact that DTB-MAC relies on a random access protocol under low densities, while at high densities DTB-MAC relies on token passing instead. Therefore, under high densities, the token circulates faster and with lower loss probability since a large number of nodes is waiting for the token and have beacons ready to transmit.

One of the token passing characteristics, which is expected to be a problem when attempting to provide efficient communication solutions in vehicular environments, is the time

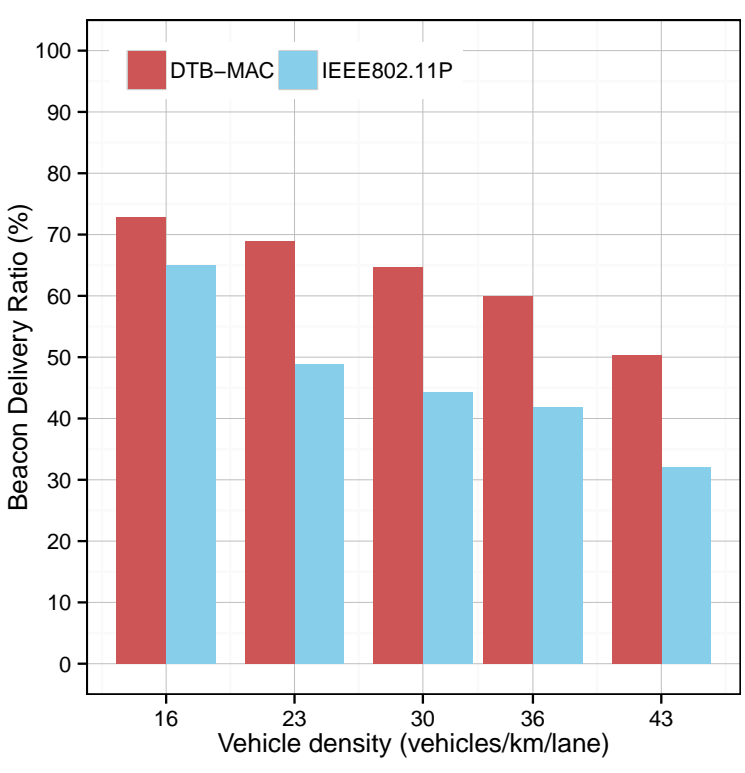

Fig. 5. Beacon delivery ratio for the highway scenario.

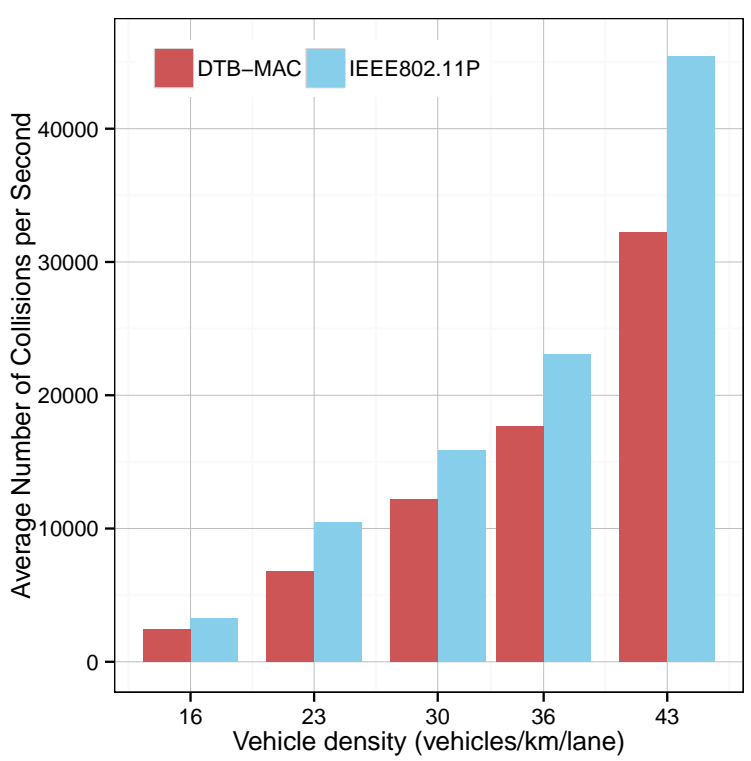

Fig. 6. Average number of collisions per second for the highway scenario.

period that each node has to wait to receive the token. With respect to the strict delay limit of safety communications, the waiting time can cause a large number of packet drops. However, Figure 7, which shows the dropped beacon ratio, can be used to prove that this waiting time can be neglected and does not have a perceptible effect on performance. As shown, the average dropped beacon ratio is less than 0.5 and, even under high network densities, it is still less than 2, thus not affecting DTB-MAC performance significantly.

We now analyse how these two protocols use the channel for beacon transmission. As expected, the DTB-MAC protocol is able to use the channel more efficient than IEEE 802.11p. IEEE $802.11 \mathrm{p}$ sends beacons by relying on a back-off pro- 
cedure, which causes lots of collisions with increasing node density. On the contrary, DTB-MAC uses token passing, which does not cause collision occurrence between neighbouring nodes if the token circulation is done correctly. Therefore, as can be seen in Figure 8, with increasing network density, DTBMAC decreases the number of collisions and increases channel usage associated to successful transmissions, thus achieving a clear improvement in successful channel utilization. We notice that, although token passing is prone to cause high delays in dynamic networks, the way DTB-MAC uses the token passing mechanism does not produce more idle time compared to IEEE $802.11 \mathrm{p}$, and is even able to improve it for some network densities.

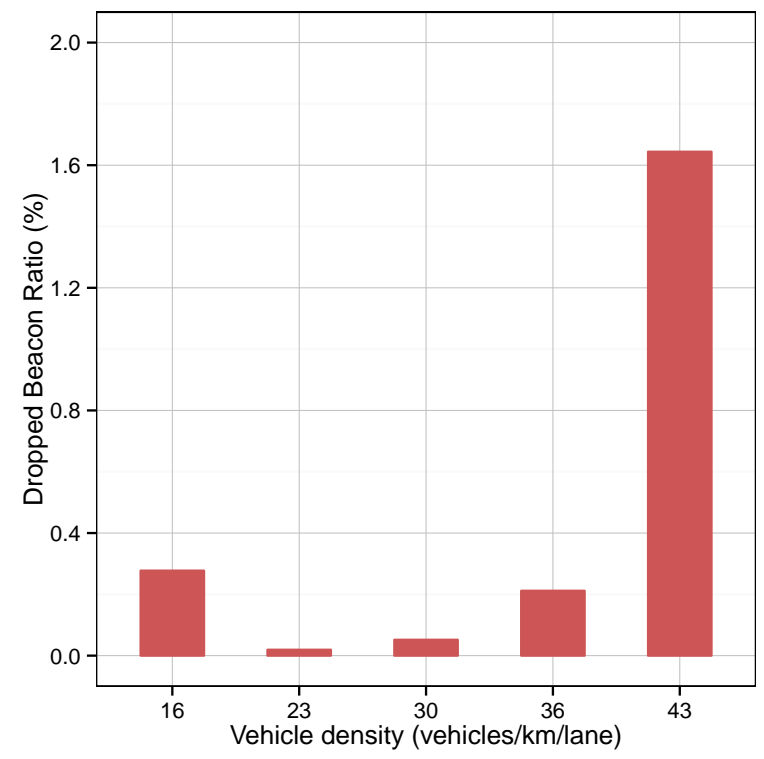

Fig. 7. Dropped beacon ratio for the highway scenario.

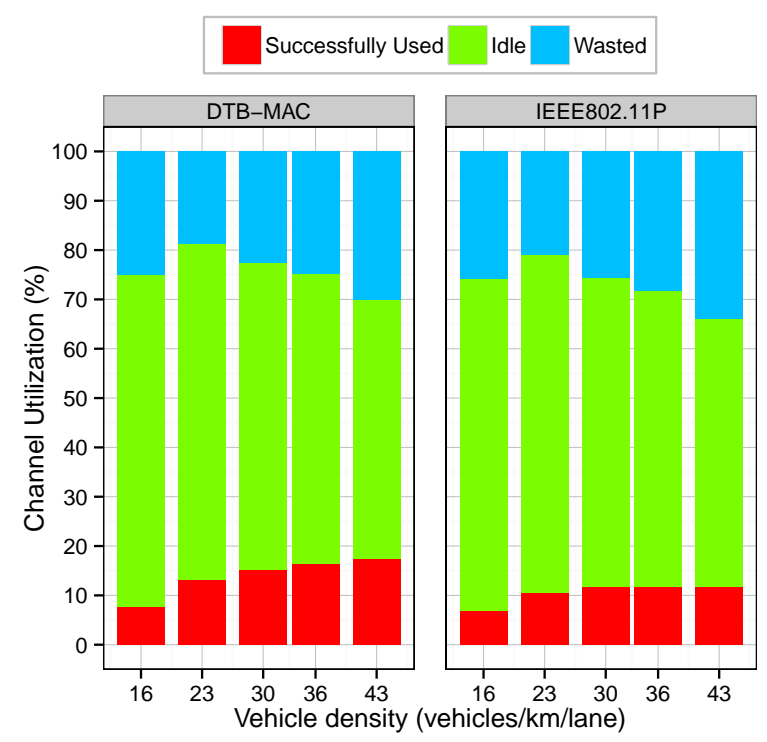

Fig. 8. Channel utilization for the highway scenario.

\section{CONCLUSION}

VANETs were developed to provide safety and comfort for car passengers by allowing vehicles to have awareness about their neighbourhood through message broadcasting. Therefore, reliability assurance of this type of message transmissions becomes very challenging because they have a direct relevance on the car passengers' safety. In this paper, we have proposed the DTB-MAC protocol, which uses a combination of a token passing mechanism and a random access MAC protocol to benefit from their advantages, being able to broadcast beacons in a reliable and efficient manner for different network densities. DTB-MAC tries to circulate a token between the ring members in order to access to the channel as soon as possible. In this way, DTB-MAC decreases the channel access collisions and improves the beacon delivery ratio. Moreover, when token passing is not possible due to the high node mobility and topology changes in the VANET, DTB-MAC uses a random access MAC protocol to provide again a situation for circulating the token in the ring. Our simulation studies show that DTB-MAC improves the beacon delivery ratio under different network densities; in particular, at high densities, the improvement ratio achieved is greater than $60 \%$.

The future contributions will be as follows: (a) propose an analytical analysis to evaluate the DTB-MAC performance; (b) evaluate the DTB-MAC protocol performance through simulation studies in urban scenarios; (c) enhance the DTBMAC protocol for RSU-based vehicular environments; and (d) extend the DTB-MAC protocol to support emergency messages.

\section{ACKNOWLEDGMENT}

This work was partially supported by the Ministerio de Ciencia e Innovación, Spain, under Grant TIN2011-27543C03-01.

\section{REFERENCES}

[1] "Comesafety: D31 european its communication architecture," Information Society Technologies, Tech. Rep., 2008.

[2] "Ieee standard for information technology- local and metropolitan area networks- specific requirements- part 11: Wireless lan medium access control (mac) and physical layer (phy) specifications amendment 6: Wireless access in vehicular environments," IEEE Std 802.11p2010 (Amendment to IEEE Std 802.11-2007 as amended by IEEE Std 802.11k-2008, IEEE Std 802.11r-2008, IEEE Std 802.11y-2008, IEEE Std 802.11n-2009, and IEEE Std 802.11w-2009), pp. 1-51, July 2010.

[3] J. Kenney, "Dedicated short-range communications (dsrc) standards in the united states," Proceedings of the IEEE, vol. 99, no. 7, pp. 11621182, July 2011.

[4] Y. Yao, L. Rao, and X. Liu, "Performance and reliability analysis of ieee 802.11p safety communication in a highway environment," Vehicular Technology, IEEE Transactions on, vol. 62, no. 9, pp. 4198-4212, Nov 2013.

[5] X. Ma, J. Zhang, and T. Wu, "Reliability analysis of one-hop safetycritical broadcast services in vanets," Vehicular Technology, IEEE Transactions on, vol. 60, no. 8, pp. 3933-3946, Oct 2011.

[6] K. Bilstrup, E. Uhlemann, E. G. Ström, and U. Bilstrup, "On the ability of the $802.11 \mathrm{p}$ mac method and stdma to support real-time vehicle-to-vehicle communication," EURASIP J. Wirel. Commun. Netw., vol. 2009, pp. 5:1-5:13, Jan. 2009. [Online]. Available: http://dx.doi.org/10.1155/2009/902414

[7] F. Borgonovo, A. Capone, M. Cesana, and L. Fratta, "Adhoc: a new, flexible and reliable mac architecture for ad-hoc networks," in Wireless Communications and Networking, 2003. WCNC 2003. 2003 IEEE, vol. 2, March 2003, pp. 965-970 vol.2. 
[8] H. Omar, W. Zhuang, and L. Li, "Vemac: A tdma-based mac protocol for reliable broadcast in vanets," Mobile Computing, IEEE Transactions on, vol. 12, no. 9, pp. 1724-1736, Sept 2013.

[9] G. Abdalla, M. Abu-Rgheff, and S. M. Senouci, "Space-orthogonal frequency-time medium access control (soft mac) for vanet," in Information Infrastructure Symposium, 2009. GIIS '09. Global, June 2009, pp. $1-8$.

[10] Y.-C. Lai, P. Lin, W. Liao, and C.-M. Chen, "A region-based clustering mechanism for channel access in vehicular ad hoc networks," Selected Areas in Communications, IEEE Journal on, vol. 29, no. 1, pp. 83-93, January 2011.

[11] K. Bilstrup, E. Uhlemann, and E. Strom, "Scalability issues of the mac methods stdma and csma of ieee 802.11p when used in vanets," in Communications Workshops (ICC), 2010 IEEE International Conference on, May 2010, pp. 1-5.

[12] K. Sjoberg, E. Uhlemann, and E. Strom, "Delay and interference comparison of csma and self-organizing tdma when used in vanets," in Wireless Communications and Mobile Computing Conference (IWCMC), 2011 7th International, July 2011, pp. 1488-1493.

[13] R. Stanica, E. Chaput, and A.-L. Beylot, "Comparison of csma and tdma for a heartbeat vanet application," in Communications (ICC), 2010 IEEE International Conference on, May 2010, pp. 1-5.

[14] A. Alonso and C. Mecklenbrauker, "Stabilization time comparison of csma and self-organizing tdma for different channel loads in vanets," in ITS Telecommunications (ITST), 2012 12th International Conference on, Nov 2012, pp. 300-305.

[15] M. Ergen, D. Lee, R. Sengupta, and P. Varaiya, "Wtrp - wireless token ring protocol," Vehicular Technology, IEEE Transactions on, vol. 53, no. 6, pp. 1863-1881, Nov 2004.

[16] S. Xianpu, Z. Yanling, and L. Jiandong, "Wireless dynamic token protocol for manet," in Parallel Processing Workshops, 2007. ICPPW 2007. International Conference on, Sept 2007, pp. 5-5.

[17] P. Wang and W. Zhuang, "A token-based scheduling scheme for wlans supporting voice/data traffic and its performance analysis," Wireless Communications, IEEE Transactions on, vol. 7, no. 5, pp. 1708-1718, May 2008.

[18] J. Zhang, K.-H. Liu, and X. Shen, "A novel overlay token ring protocol for inter-vehicle communication," in Communications, 2008. ICC '08. IEEE International Conference on, May 2008, pp. 4904-4909.

[19] Y. Bi, K.-H. Liu, L. Cai, X. Shen, and H. Zhao, "A multi-channel token ring protocol for qos provisioning in inter-vehicle communications," Wireless Communications, IEEE Transactions on, vol. 8, no. 11, pp. 5621-5631, November 2009.

[20] L. E. J. Behrisch, M. Bieker and D. Krajzewicz, "Sumo - simulation of urban mobility: An overview in: Simul 2011," in The Third International Conference on Advances in System Simulation, 2011.

[21] "Omnet++ home page," http://www.omnetpp.org, accessed: 2014-02-30

[22] C. Sommer, R. German, and F. Dressler, "Bidirectionally coupled network and road traffic simulation for improved ivc analysis," Mobile Computing, IEEE Transactions on, vol. 10, no. 1, pp. 3-15, Jan 2011.

[23] C. Sommer, D. Eckhoff, and F. Dressler, "Ivc in cities: Signal attenuation by buildings and how parked cars can improve the situation," Mobile Computing, IEEE Transactions on, vol. PP, no. 99, pp. 1-1, 2013.

[24] H. Song and H. S. Lee, "A survey on how to solve a decentralized congestion control problem for periodic beacon broadcast in vehicular safety communications," in Advanced Communication Technology (ICACT), 2013 15th International Conference on, Jan 2013, pp. 649654. 